\title{
ALKYL AMMONIUM INTERCALATION OF MOZAMBICAN BENTONITE
}

\section{Pedro H. Massinga Jr. ${ }^{1}$, Walter W. Focke*, Phillip L. de Vaal, Maria Atanasova ${ }^{2}$}

Institute of Applied Materials, Department of Chemical Engineering, University of Pretoria, phone: (+27) 12420 4398, Fax: (+27) 12420 2516, Pretoria 0002, South Africa

ABSTRACT: Organo-bentonites containing quaternary ammonium surfactants were prepared using purified bentonite from the Boane deposit in Mozambique. The intercalation was effected by intimate mixing of the surfactants into a concentrated dispersion of soda ash activated bentonite. TG indicated organic contents that exceeded CEC expectations by up to 21 mass \%. XRD results were consistent with: (i) paraffin-type extended chain intercalation at tilt angles of $27^{\circ}$ to $33^{\circ}$; and (ii) interdigitated monolayer intercalation of the C14 single alkyl chain surfactant and bilayer intercalation of the C16 double alkyl chains and its 1:1 mixture with the single chain surfactant. However, FTIR analysis revealed disordered alkyl chain conformations. White oil dispersions containing 10 mass \% organo-bentonite (inorganic basis) featured high viscosities. They showed strong shear thinning behaviour confirming the ability of the montmorillonite particles to exfoliate.

Keywords: Intercalation, bentonite, quaternary ammonium salt, organo-bentonite.

\footnotetext{
${ }^{*}$ Corresponding Author. Tel.: +27 12420 3728; fax: +27 124202516.

E-mail address: walter.focke@up.ac.za

${ }^{1}$ Permanent address: University Eduardo Mondlane, Faculty of Natural Sciences, Chemistry Department, P.O.Box 257, Maputo, Mozambique. E-mail address: massingajr@uem.mz

${ }^{2}$ Permanent address: Council for Geoscience, Private Bag X112, Pretoria 0001, South Africa. E-mail address: mtg@geoscience.org.za
} 


\section{INTRODUCTION}

Well-dispersed nanoparticulate mineral particles can enhance properties of polymeric materials such as strength and stiffness, dimensional stability, flame retardancy, gas barrier properties, and UV stability (Ahmadi et al., 2005; Alexandre and Dubois, 2000; Fischer, 2003; LeBaron et al., 1999; Lee and Lee, 2004; Patel et al., 2007; Ray and Okamoto, 2003; Wang et al., 2004). The natural abundance of clay minerals in nearly pure mineralogical form, coupled with their inherently low processing costs, promises widespread future applications in the automotive, electronics, food packaging and biotechnology industries (Konta, 1995; Rehab and Salahuddin, 2005; Wang et al., 2004).

Individual montmorillonite (MMT) layers are about $0.96 \mathrm{~nm}$ thick and they have lateral dimensions that are more than two orders of magnitude larger (Paul et al., 2005; Utracki, 2004; Vaccari, 1998). The high aspect ratio of the clay particles is an important factor in polymer property enhancement. Exfoliating MMT layers in a polymer matrix leads to a very large interfacial surface area for interaction with the polymer chains. Delamination of individual clay mineral layers into polymer matrix is doubtful (Pavlidou and Papaspyrides, 2008). However, exfoliation can lead to a uniform dispersion of particles corresponding to stacks comprising a few clay mineral layers. This is a key requirement for improving mechanical and barrier properties of these nanocomposites (Chaiko and Leyva, 2005; Gopakumar et al., 2002; LeBaron et al., 1999; Powell and Beall 2006; Yang et al., 1999). Dispersion of neat clay particles is difficult owing to an intrinsic incompatibility between the hydrophobic polymer matrix and the hydrophilic clay mineral surfaces. Better matching of the surface polarities can be achieved by appropriate organic modification of the clays. This is usually accomplished via ion exchange reactions (de Paiva et al., 2008). The inorganic cations in the clay interlayer space, e.g. $\mathrm{Ca}^{2+}$ or $\mathrm{Na}^{+}$, can directly be exchanged with organic cations by means of an intercalation process. The substitution with organic cations can be partial or 
complete, and in some cases it may even exceed the cation exchange capacity (CEC) (Utracki, 2004; Zhu et al., 2008). Intercalation of organic cations is an important first step in the preparation of clay mineral/polymer hybrids. It modifies the clay surface chemistry, changing its nature from hydrophilic to organophilic. This renders the MMT compatible with organic polymers and facilitates the exfoliation of the clay mineral layers in the polymer matrix (Lee and Lee, 2004; Powell and Beall, 2006).

Quaternary alkylammonium salts (bromides or chlorides) are the organic cations most often intercalated into MMT's. Long chain quaternary ammonium ions are preferred as they lead to a larger interlayer spacing. This allows facile penetration of polymer chains into the clay interlayer space. The co-intercalation of polymer chains ultimately aids dispersions of distinct MMT particles throughout the polymer matrix.

The objective of this study was to render bentonite from the Boane deposit in Mozambique suitable for the preparation of bentonite-based polymer nanocomposites. Towards this purpose, intercalation of single chain and double chain quaternary ammonium ions, as well as a mixture of these two surfactants, was explored. Boane bentonite features a very light colour but contains significant amounts of cristobalite that proved recalcitrant to attempts for removal by a variety of purification methods. The effect of this impurity on performance was assessed with rheometry and abrasion testing of white oil dispersions of the organobentonites.

\section{MATERIALS AND METHODS}

The bentonites used in this investigation were from the Boane deposit in Mozambique and from the Koppies mine in South Africa. G \& W Base and Industrial Minerals supplied samples of the crude bentonites in milled powder form and in purified form as sodium-activated dispersions with an off-white colour (Boane) or a light brown colour (Koppies). The cation exchange capacities of the bentonites were determined by the supplier as $0.70 \mathrm{meq} / \mathrm{g}$ and $0.85 \mathrm{meq} / \mathrm{g}$ respectively using 
the methylene blue method (Hang and Brindley, 1970). The slurry $\mathrm{pH}$ for the Boane bentonite was $\mathrm{pH}=7.4$ and the solids content was 19.2 mass \%. The corresponding values for the Koppies bentonite slurry were $\mathrm{pH}=9.9$ and 18.9 mass $\%$ solids. This communication provides details on the properties of the Boane bentonite and its modifications. Koppies bentonite showed very similar intercalation behaviour that will be reported elsewhere. However, modified Koppies bentonite provided a convenient reference material for assessing the effect of impurities in the Boane bentonite on abrasion behaviour.

The cationic surfactant Varisoft 432 CG is a product of Degussa (Goldschmidt GmbH). It is a double chain quaternary ammonium ions with the chain lengths varying from twelve to eighteen carbons but it contains mostly N,N'-dihexadecyl dimethylammonium chloride. The single chain surfactant used was tetradecyl trimethylammonium bromide (Cetrimide BP, Purum grade, ex FeF Chemicals A/S, Copenhagen). Intercalation experiments were conducted using either the single (SC14), the double (DC16) chain quaternary ammonium ions or a ca. 1:1 mixture (SC14 + DC16), molar basis. In these calculations it was assumed that the cationic surfactant DC16 (Varisoft 432 CG) comprised pure N,N’-dihexadecyl dimethylammonium chloride. The intercalation experiments were performed using a fifty percent excess of the surfactants based on the estimated bentonite CEC. A typical intercalation experiment was performed as follows: The soda ash activated bentonite dispersion was placed in a planetary mixer. The surfactant solution or powder was added while mixing. Mixing was continued intermittently for about five hours. The intercalated bentonite was recovered by centrifugation, washed repeatedly with water until halide ions could not be detected with a $1 \mathrm{M}$ silver nitrate solution. The solids were dried at ambient temperature and humidity. They were crushed and milled into a fine powder using a mortar and pestle. Multiple instrumental techniques were used to characterize and examine the properties of the bentonite samples before and after organic treatment. 
Elemental composition was determined by X-ray fluorescence (XRF). The intercalated materials were ashed before analysis in order to reduce their bulk. The samples were ground to $<75 \mu \mathrm{m}$ in a tungsten carbide mill and roasted at $1000^{\circ} \mathrm{C}$. Then $1 \mathrm{~g}$ sample was added to 9 $\mathrm{g} \mathrm{Li}_{2} \mathrm{~B}_{4} \mathrm{O}_{7}$ and fused into a glassed bead. Major element analysis was executed on the fused bead using an ARL9400XP+ spectrometer.

Thermal analysis was performed using the dynamic method on a Mettler Toledo A851 TGA/SDTA instrument. About 15 mg powder was placed in open $70 \mu \mathrm{L}$ alumina pans. Temperature was scanned from 25 to $1000{ }^{\circ} \mathrm{C}$ at a rate of $10{ }^{\circ} \mathrm{C} / \mathrm{min}$ with air flowing at a rate of $50 \mathrm{~mL} / \mathrm{min}$.

Mineral identification and composition assessment of pristine bentonite was performed by the South African Council for Geoscience. The XRD instrument used was a BRUKER D8 Advance diffractometer using $\mathrm{Cu} \mathrm{K} \alpha$ radiation $(\lambda=0.15406 \mathrm{~nm})$. The system featured a Johansson crystal primary monochromator and LynxEye detector with $3.7^{\circ}$ active area. XRD patterns were recorded for random powder preparations. The mineral identification was based on a BRUKER DIFFRAC ${ }^{\text {Plus }}$ - EVA evaluation program. The phase concentrations were determined by Rietveld quantitative analysis with DIFFRAC ${ }^{\text {Plus }}$ - TOPAS software to an accuracy of $\pm 1 \%$.

$\mathrm{XRD}$ analysis of random powder samples of the organo-bentonites was performed inhouse on a PANalytical X-pert Pro machine. The instrument featured variable divergence and receiving slits and an X'celerator detector using Fe filtered Co K $\alpha$ radiation $(\lambda=0.17901 \mathrm{~nm})$. X'Pert High Score Plus software was used for data reduction.

IR spectra were recorded on a Perkin Elmer RX I FT-IR spectrometer. Approximately 2 mg of dry sample was combined with $100 \mathrm{mg}$ of spectroscopic grade $\mathrm{KBr}$ and ground into a fine powder. The mixture was pressed into a $13 \mathrm{~mm} \phi$ die pellet. The reported spectra 
represent averages of 32 scans at a resolution of $2 \mathrm{~cm}^{-1}$. The averaged data was backgroundcorrected using a pure $\mathrm{KBr}$ pellet.

Surface morphology was examined by low resolution scanning electron microscopy (SEM) using a JEOL 840 instrument. The powder samples were coated five times with gold using a SEM Polaron E5200. A SEM fitted with an Energy Dispersive X-Ray analyzer (SEM/EDS) was used to examine the morphological and mineralogical association of montmorillonite and cristobalite. A Leica Stereoscan 440 instrument linked to an OXFORD INCA EDS with Oxford SDD detector was used. Polished stubs of the samples were prepared and coated with carbon for conductivity using an EMITECH K950X sputter coater. In addition, an Oberkochen Zeiss Ultra Plus microscope was used for field emission scanning electron microscopy (FESEM).

Rheological studies were performed on white oil thickened with 10 mass \% organobentonite (inorganic basis). Akulu Marchon supplied white oil with a density of $0.831 \mathrm{~g} / \mathrm{cm}^{3}$ at $20^{\circ} \mathrm{C}$ and a viscosity of $13.8 \mathrm{mPa} . \mathrm{s}$ at $40^{\circ} \mathrm{C}$. The propensity of the organo-bentonite particles to exfoliate was probed by dispersing them under high shear in white oil in the presence of a polar activator. The powders were thoroughly mixed into the white oil liquid using an agate mortar and pestle. An amount, equivalent to 5 mass \% of the clay, of a polar activator mixture (comprising $95 \%$ ethanol and $5 \%$ water) was added and mixing was continued until a homogeneous paste was obtained. The viscosities of the white oil dispersions were determined at $30{ }^{\circ} \mathrm{C}$ on an Anton Paar Physica UDS 200 rheometer using the cone-and-plate geometry. The gap was set at $0.51 \mathrm{~mm}$ and the diameter of the disc was 50 $\mathrm{mm}$.

Abrasion testing was performed using the High-Frequency Reciprocating Rig (HFRR apparatus) according to the test procedures described in ISO 12156. Dispersions containing 10 mass \% organo-bentonite in white oil were used for this purpose. A $2 \mathrm{~mL}$ sample of the 
fluid under test was placed in a reservoir maintained at a temperature of $25^{\circ} \mathrm{C}$. A fixed steel ball was held in a vertically mounted chuck and forced against a horizontally mounted stationary steel disk at an applied load of $2 \mathrm{~N}$. The test ball was oscillated at a fixed frequency of $50 \mathrm{~Hz}$ and a stroke length of $1 \mathrm{~mm}$ while the contact interface was fully immersed in the fluid reservoir. The test duration was 75 min.

The stationary, bottom specimen was manufactured from AISI E-52100 steel, with a Vickers hardness "HV 30" scale number of 190 to 210, lapped and polished to a surface finish of $\mathrm{Ra}<0.02 \mu \mathrm{m}$. The top specimen, which was oscillated against the bottom disk, was a sphere, 6 mm in diameter, manufactured from grade 28 AISI E-52100 steel. It had a Rockwell hardness "C" scale (HRC) number of 58 to 66 and a surface finish of $\mathrm{Ra}<0.05 \mu \mathrm{m}$.

On completion of the test, the disk and ball were cleaned and a microscope photograph of the wear scar under 100X magnification on the ball was taken. The wear scar diameter was estimated as the average of the wear scar measurements in the X-direction and the $\mathrm{Y}$ direction. In the ISO 12156 test, the average wear scar diameter is taken as a measure of the lubricity of the fluid under test. In the present case, the wear scar diameter provided an indication, on a comparative scale, of the extent of abrasive wear. The average friction coefficient measured over the duration of the test was also recorded.

\section{RESULTS AND DISCUSSION}

\section{Appearance, composition and thermal stability}

The Rietveld quantitative analysis of the XRD data indicated that the crude Boane bentonite contained 60.3 mass \% montmorillonite, 35.7 mass \% cristobalite and 4.0 mass \% quartz. Table 1 presents the chemical composition of the crude Boane bentonite and the ash from organically treated bentonite samples as determined by X-Ray fluorescence (XRF). The high silica content was attributed to the crystobalite present as a major impurity. The data showed 
substantial reduction in the $\mathrm{Na}$ and $\mathrm{Ca}$ content following organic modification. This provided evidence for the successful intercalation of the organic cations, i.e. that they replaced the $\mathrm{Na}^{+}$ and $\mathrm{Ca}^{2+}$ in the pristine bentonites by ion exchange.

The low resolution SEM micrographs (not shown here) revealed flake-shape particles. The aggregates of the crude bentonite appeared significantly larger than those for the organically modified samples. Fig. 1 shows SEM/EDS images of a SC14 organically modified sample embedded in an epoxy resin. The backscattered electron images showed a large region comprising a $\mathrm{SiO}_{2}$ phase. The multiple cracks through this cristobalite-containing region suggested that it comprised a large agglomerate of much finer particles rather than a single large particle. This conjecture was confirmed by the FESEM images shown in Fig. 2. They showed the presence of rounded nano-sized particles on the surfaces of the clay mineral particles and also in aggregated form. Most of these particles were much smaller than $100 \mathrm{~nm}$ in size. The large aggregate shown in Fig. 1 was attributed to the surface energy mismatch between the uncoated silica nanoparticles and the surfactant coated bentonite flakes.

Fig. 3 shows mass loss curves for the crude and organically treated bentonites. Mass loss proceeded stepwise in all samples and tended to increase with increasing organic content. Only two major thermal events were observed in the TG curve of the crude bentonite. The TG curves of the organo-bentonites showed additional thermal events.

The endothermic peak displayed below $150{ }^{\circ} \mathrm{C}$ in the DTA curve for the raw bentonite was attributed to the loss interlayer water (Fig. 4). The mass loss associated with the dehydration event occurring below $150{ }^{\circ} \mathrm{C}$ amounted to 16.6 mass \% for the crude bentonite and less than 3 mass \% for the organo-bentonites. The higher water content of the crude bentonite was expected as the $\mathrm{Ca}^{2+}$ and $\mathrm{Na}^{+}$ions probably have a greater tendency for hydration than the oleophilic surfactant.

The DTA curve for the crude bentonite is almost featureless in the region between $150{ }^{\circ} \mathrm{C}$ and $600{ }^{\circ} \mathrm{C}$ (Fig. 4). In contrast, the curves obtained for the organo-bentonites showed significant 
exothermic events in this temperature range. These were attributed to the oxidative decomposition of the intercalated organic species in the air atmosphere (Hedley et al., 2007; Tiwari et al., 2008). The SC14 single chain surfactant featured a broad exotherm peak centred at ca. $330{ }^{\circ} \mathrm{C}$. The DC16 surfactant had a lower thermal stability. A well-defined exotherm onset was observed just below $200{ }^{\circ} \mathrm{C}$ and was followed by a second broader peak centred at ca. $370{ }^{\circ} \mathrm{C}$. The bentonite intercalated by a mixture of these two surfactants showed behaviour consistent with independent degradation of the two surfactants present. According to Xie et al. (2001), the decomposition onset temperatures of alkyl ammonium organoclays are essentially independent of the alkyl chain length. However, the current results clearly indicated a lower thermal stability of the double chain surfactant-containing systems compared to the single chain intercalated bentonite.

The inorganic content of dry samples, presented in Table 2, was estimated from the residual mass observed at $1000{ }^{\circ} \mathrm{C}$ relative to that measured at $150{ }^{\circ} \mathrm{C}$. The organic content of the intercalated bentonites was determined by comparing their mass loss values with that obtained for the neat bentonite. These values were compared with the theoretical organic content calculated on the basis of complete ion exchange equivalent to the CEC (Table 2). In all cases the surfactants intercalated to a level that exceeded the CEC theoretical expectation by $10 \%, 11 \%$ and $21 \%$ for the SC14, DC16 and the surfactant mixture intercalated bentonites respectively.

\section{State of the intercalated alkylammonium ions}

Organic cations introduced up to the CEC level by ion exchange (Lagaly, 1986) are electrostatically “attached” to the clay mineral surfaces. The "excess” surfactant molecules are intercalated in their neutral salt form, i.e. together with their (hydrated) counterions (Klapyta et al., 2001; Lagaly, 1986). The incorporation of excess surfactant is driven by van der Waals interactions. Dense close-packing of the surfactants in the interlayer space improves the van der Waals interactions between long alkyl chains (Calderon et al., 2008; 
Janek and Lagaly, 2003; Kwolek et al., 2003; Pan et al., 1997; Tahani et al., 1999; Yui et al., 2002). Denser packing also requires better charge shielding of the same-charge surfactant head groups. Close packing of long-chain surfactants can be facilitated by single-layer interdigitated intercalation, i.e. where the organic molecules are placed in a way that the polar heads are in opposite directions in order to minimize electrostatic repulsion between these head groups.

Powder XRD patterns for crude and organically modified bentonite samples are presented in Fig. 5. The crude sample showed a montmorillonite basal reflection at $2 \theta=5.88^{\circ}$ corresponding to $\mathrm{d}_{001}=1.50 \mathrm{~nm}$. This falls within the range of expected values $(1.2$ to $1.6 \mathrm{~nm})$ for typical smectite minerals observed under ambient temperature and humidity (Utracki, 2004). Montmorillonite was identified by the $d_{001}$ value coupled with $d_{060}=0.15 \mathrm{~nm}$ and the Greene-Kelly test. The test consisted of Li-saturation, glycolation and heating to $300{ }^{\circ} \mathrm{C}$. After this treatment, the basal spacing collapsed to $0.96 \mathrm{~nm}$ indicating montmorillonite (Greene-Kelly, 1955). Upon ethylene glycol saturation for $24 \mathrm{~h}$, the $\mathrm{d}_{001}$ expanded to $1.72 \mathrm{~nm}$ and collapsed to $1.03 \mathrm{~nm}$ after heating for $1 \mathrm{~h}$ at $550^{\circ} \mathrm{C}$ (Brown and Brindley, 1980). Reflections characteristic for $\alpha$-cristobalite were the following: $\mathrm{d}_{101}=0.40 \mathrm{~nm}, \mathrm{~d}_{102}=0.28 \mathrm{~nm}, \mathrm{~d}_{200}=0.25 \mathrm{~nm}, \mathrm{~d}_{301}=$ $0.16 \mathrm{~nm}$ and $\mathrm{d}_{302}=0.15 \mathrm{~nm}$ (Guven and Grim, 1972).

The single chain quaternary ammonium ions contained 14 carbon atoms and the double chain quaternary ammonium ions contained 16 carbon atoms.

The linear alkyl substituents of the intercalated quaternary ammonium ions may assume paraffin-like extended chain conformations. In this case space-filled packing is facilitated when the chains are tilted at an angle $\alpha$ with respect to the plane of the clay mineral layers (He et al., 2006; Lagaly, 1986; Lagaly and Weiss, 1970a; Lagaly and Weiss, 1970b). The expected basal spacings for interdigitated monolayer intercalation and bilayer intercalation are given by the following approximate expressions (Kopka, 1998; Lagaly and Weiss, 1970b): 
Monolayer:

$$
d_{L}=1.18+0.127 n_{C} \sin \alpha
$$

Bilayer:

$$
d_{L}=1.66+0.254 n_{C} \sin \alpha
$$

where $d_{L}$ is the basal spacing, $n_{C}$ is the number of carbon atoms in the alkyl chain and $\alpha$ is the tilt angle.

The values indicated for the constants in these equations are only approximate. They take into account the following contributions (Lagaly and Weiss, 1970a; Lagaly and Weiss, 1970b): the thickness of the bentonite layer $(0.66 \mathrm{~nm})$; the van der Waals radius of surface oxygen atoms $(0.14$ $\mathrm{nm})$; the van der Waals radius of a methyl group $(0.20 \mathrm{~nm})$; the distance between a terminal methyl group and an oxygen atom $(0.12 \mathrm{~nm})$; the projected length of the $\mathrm{C}-\mathrm{N}$ bond $(0.12 \mathrm{~nm})$; a correction accounting for the fact that methyl groups can actually penetrate the six-member oxygen rings of the silicate sheets (maximum depth $0.13 \mathrm{~nm}$ ), and finally the gap between the terminal methyl groups for the case of bilayer intercalation $(0.20 \mathrm{~nm})$. These values yield separation distances of $x \mathrm{CH}_{3}{ }^{\cdots} \mathrm{O} \approx 0.12 \mathrm{~nm}$ and $x \mathrm{~N}-\mathrm{CH}_{3}{ }^{\cdots} \mathrm{O} \approx 0.40 \mathrm{~nm}$.

The XRD measured basal spacings were $2.0 \mathrm{~nm}, 2.93 \mathrm{~nm}$ and $2.72 \mathrm{~nm}$ for the organobentonite SC14, the double chain C16 surfactant and the 1:1 mixture respectively. Tilt angles, estimated from these values and equation (1) or equation (2), were $27^{\circ}, 33^{\circ}$ and $32^{\circ}$ respectively. These angles are equivalent to about half the value expected for typical paraffin-type tilt angles (Lagaly, 1986).

It must be noted that the interlayer structure and the conformations assumed by intercalated alkyl chain surfactants, e.g. quaternary ammonium ions, has been the subject of intense debate (Lagaly, 1976; Lagaly, 1986; Vaia et al. 1994). At high surfactant loadings, two limiting cases can actually be envisioned. The first presumes, as we did above, that the chains are present in a chain extended (paraffin-type), tilted arrangement. The alternative arrangement assumes disordered chain conformations containing numerous gauche conformers (Lagaly, 1976). Both can be consistent with the basal spacings observed by XRD. 
These two different arrangements, although indistinguishable by X-ray investigation, nevertheless constitute very different interlayer structures and molecular environments (Vaia et al., 1994).

FTIR spectra for the organo-bentonites are shown in Fig. 6. They showed strong bands near $2920 \mathrm{~cm}^{-1}$ and $2850 \mathrm{~cm}^{-1}$. These arise from the $\mathrm{CH}_{2}$ asymmetric $v_{\mathrm{as}}\left(\mathrm{CH}_{2}\right)$, and symmetric $v_{\mathrm{s}}\left(\mathrm{CH}_{2}\right)$ stretch modes, respectively. Vaia et al. (1994) proposed the use of the $v_{\text {as }}\left(\mathrm{CH}_{2}\right)$ band to probe the nature of the interlayer structure. The wavenumber and the width of this band are very sensitive to the gauche/trans conformer ratio and the packing density of the chain methylenes. It varies from $2917.8 \mathrm{~cm}^{-1}$ for the methylene chains in the all-trans ordered state in a crystalline surfactant to $2928.9 \mathrm{~cm}^{-1}$ when the chains are in a liquid-like environment (Hongping et al., 2004; Vaia et al., 1994). The peak positions of the methylene asymmetric bands found for the present organo-bentonites are presented in Table 3. The observed values for the intercalated bentonites were similar and located in the middle of the range indicating that the chains adopted a more disordered, liquid-like structure. Such trans-gauche disorder promotes dense packing of the chains and leads to improved surface mating between the terminal methyl groups and the silicate layers (Lagaly, 1976).

\section{Rheology: Probing the propensity for exfoliation}

Organoclays are well-known rheology modifiers (Jones, 1983). They are widely used as thickeners and to control the thixotropic properties of organic systems such as paints and greases. These properties rely on the exfoliation of the clay mineral particles and a three dimensional assembly into mesoscale card-house structures. Water, carried in by a suitable chemical activator, migrates in between the hydroxyl groups on adjacent clay particle edges. It forms hydrogen bond bridges between the hydroxyl groups helping to develop and stabilize 
the gel structure. Application of high shear rates or prolonged action of lower shear forces causes progressive parallel alignment of the platelets leading to the observation of pronounced shear thinning (Wagener and Reisinger, 2003). In fact, it was proposed that the intensity of this effect can be used to characterize the degree of exfoliation of clay mineral particles in a polymer matrix (Wagener and Reisinger, 2003) or in wax melts (Wang et al., 2008).

The effect of the shear rate on the viscosity, measured at $30^{\circ} \mathrm{C}$ for dispersions containing 10 mass \% organo-bentonite (inorganic basis), is presented in Fig. 7. The viscosity of the white oil showed Newtonian behaviour, i.e. it was independent of the shear rate. In contrast, the organo-bentonite dispersions showed strong shear thinning. The apparent viscosities showed a nearly linear decrease with increase in shear rate on the log - log plot (Fig. 7). The slope of this plot corresponds to the shear thinning exponent $n$ of the power law viscosity equation (Wagener and Reisinger, 2003). For the present bentonites, the values of the shear thinning exponents were about $n=-3 / 4$ and this high value confirmed the propensity of the present organo-bentonites to exfoliate in a suitable organic medium.

\section{Abrasion testing}

The HFRR wear and dynamic friction test results are presented in Table 4. Only one run was performed in each case with the exception of the Boane (SC14 + DC 16) sample for which a duplicate run was recorded. The results should therefore be regarded as indicative only as the repeatability of the wear scar measurements is only ca. $63 \mu \mathrm{m}$. The neat white oil acted like a good lubricant featuring a low friction coefficient. Visual inspection of the wear scar revealed limited wear and a scar diameter of only $165 \mu \mathrm{m}$. The friction coefficient was about twice as large for the organo-bentonite samples. The wear scar diameter was also much larger than that obtained with the white oil. Visual inspection of the wear scars confirmed that abrasive wear had taken place. In contrast to the Boane bentonite, the purified Koppies bentonite contains negligible amounts of silica or cristobalite impurities. Nevertheless it showed wear behaviour 
similar to the corresponding Boane (SC14 + DC16) modified bentonite. This suggests that the abrasive properties derived primarily from the bentonite itself and that the cristobalite impurity probably did not contribute significantly to increased abrasiveness of the Boane bentonite. This was expected in view of the extreme fineness of the particles constituting this impurity as revealed by the FESEM pictures.

\section{CONCLUSIONS}

Organo-bentonites were prepared using purified bentonite from the Boane deposit in Mozambique. This bentonite contains significant amounts of cristobalite that can not be removed cost effectively. Quaternary ammonium surfactants were intercalated using soda ash activated bentonite dispersions. TG indicated organic contents that exceeded CEC expectations by 10 to $21 \%$. XRD results were consistent with: (i) paraffin-type extended chain intercalation at tilt angles of $27^{\circ}$ to $32^{\circ}$; and (ii) interdigitated monolayer intercalation of the C14 single alkyl chain surfactant and bilayer intercalation of the C16 double alkyl chains and its 1:1 mixture with the single chain surfactant. However, FTIR analysis indicated disordered chain conformations. White oil dispersions containing 10 mass \% organo-bentonite (inorganic basis) showed high viscosities and strong shear thinning confirming the ability of the clay mineral particles to exfoliate. These results suggest that it may not be necessary to remove the recalcitrant cristobalite impurity phase.

\section{ACKNOWLEDGEMENTS}

Financial support for this research from the Institutional Research Development Programme (IRDP) and the South Africa/Mozambique Collaboration Programme of the National Research Foundation (NRF) is gratefully acknowledged. We are also grateful for samples and technical support provided by G \& W Base \& Industrial Minerals. The authors also acknowledge useful suggestions by Prof. Dr. Lagaly with respect to the estimation of basal spacing. 


\section{REFERENCES}

Ahmadi, S., Huang, Y., Li, W., 2005. Fabrication and physical properties of EPDM organoclay nanocomposites, Compos. Sci. Technol. 65, 1069-1076.

Alexandre, M., Dubois, P., 2000. Polymer-layered silicate nanocomposites: preparation, properties and uses of a new class of materials, Mater. Sci. Eng. 28, 1-63.

Brown, G., Brindley, G., 1980. Crystal structure of clay minerals and their X-ray identification, Mineralogical Society, London, Monograph 5, 305-360.

Calderon, J., Lennox, B., Kamal, M., 2008. Thermal stable phosphonium-montmorillonite organoclays, Appl. Clay Sci. 40, 90-98.

Chaiko, D., Leyva, A., 2005. Thermal transitions and barrier properties of olefinic nanocomposites, Chem. Mater. 17, 13-19.

de Paiva, L., Morales, A., Diaz, F., 2008. Organoclays: Properties, preparation and applications, Appl. Clay Sci. 42, 8-24.

Fischer, H., 2003. Polymer nanocomposites: from fundamental research to specific applications, Mater. Sci. Eng. C 23, 763-772.

Gopakumar, T., Lee, J., Kontopoulou, M., Parent, J., 2002. Influence of clay exfoliation on the physical properties of montmorillonite/polyethylene composites, Polymer 43, 54835491.

Greene-Kelly, R., 1955. Dehydration of the montmorillonite minerals. Mineral Mag. 228. 
Guven, N., Grim, R., 1972. X-Ray Diffraction and electron optical studies on smectite and $\alpha$ cristobalite associations, Clays Clay Miner. 20, 89-92.

Hang, P., Brindley, G., 1970. Methylene Blue absorption by clay minerals. Determination of surface areas and cation exchange capacities (clay-organic studies xviii), Clays Clay Miner. 18, 203-212.

He, H., Frost, R., Bostrom, T., Yuan, P., Duong, L., Yang, D., Xi, Y., Kloprogge, T., 2006. Changes in the morphology of organoclays with HDTMA + surfactant loading, Appl. Clay Sci. 31, 262-271.

Hedley, C., Yuan, G., Theng, B., 2007. Thermal analysis of montmorillonites modified with quaternary phosphonium and ammonium surfactants, Appl. Clay Sci. 35, 180-188.

Hongping, H., Ray, F., Jianxi, Z., 2004. Infrared study of HDTMA+ intercalated montmorrilonite. Spectrochim. Acta 60, 2853-2859.

Janek, M., Lagaly, G., 2003. Interaction of a cationic surfactant with bentonite: a colloid chemistry study, Colloid Polym. Sci. 281, 293-301.

Jones, T.R., 1983. The properties and uses of clays which swell in organic solvents. Clay Miner. 18, 399-410.

Klapyta, Z., Fujita, T., Iyi, N., 2001. Adsorption of dodecyl- and ctadecyltrimethylammonium ions on a smectite and synthetic micas, Appl. Clay Sci. 19, 5-10.

Konta, J., 1995. Clay and man: Clay raw materials in the service of man, Appl. Clay Sci. 10, 275-335. 
Kopka, H., Beneke, K., Lagaly, G., 1998. Anionic surfactants between double metal hydroxide layers, J. Colloid Interface Sci. 123, 427-436.

Kwolek, T., Hodorowicz, M., Stadnicka, K., Czapkiewicz, J., 2003. Adsorption isotherms of homologous alkyldimethylbenzylammonium bromides on sodium montmorillonite, J. Colloid Interface Sci. 264, 14-19.

Lagaly, G., 1976. Kink-block and gauche-block structures of bimolecular films. Angew. Chem. Int. Ed. Engl. 15, 575-586.

Lagaly, G., 1986. Interaction of alkylamines with different types of layered compounds, Solid State Ionics 22, 43-51.

Lagaly, G., Weiss, A., 1970a. Anordnung und Orientierung kationischer Tenside auf Silicatoberflächen. II. Paraffinähnliche Strukturen bei den n-AlkylammoniumSchichtsilicaten mit hoher Schichtladung (Glimmer). Kolloid-Z. u. Z. Polymere 237, 364368.

Lagaly, G., Weiss, A., 1970b. Anordnung und Orientierung kationischer Tenside auf Silicatoberflächen. III. Paraffinartige Strukturen bei den n-AlkylammoniumSchichtsilicaten mit mittlerer Schichtladung (Vermiculite). Kolloid-Z. u. Z. Polymere 238, 485-493.

LeBaron, P., Wang, Z., Pinnavaia, T., 1999. Polymer-layered silicate nanocomposites: an overview, Appl. Clay Sci. 15, 11-29. 
Lee, J., Lee, H., 2004. Characterization of organobentonite used for polymer nanocomposites, Mater. Chem. Phys. 85, 410-415.

Pan, J., Yang, G., Han, B., Yan, H., 1997. Studies on Interaction of

Dodecyltrimethylammonium Bromide with Na- and Al-Montmorillonite, J. Colloid Interface Sci. 194, 276-280

Patel, H., Somani, R., Bajaj, H., Jasra, R., 2007. Preparation and characterization of phosphonium montmorillonite with enhanced thermal stability, Appl. Clay Sci. 35, 194200.

Paul, D., Zeng, Q., Yu, A., Lu, G., 2005. The interlayer swelling and molecular packing in organoclays, J. Colloid and Interface Sci. 292, 462-468.

Pavlidou, S., Papaspyrides, C., 2008. A review on polymer-layered silicate nanocomposites, Prog. Polym. Sci. 33, 1119-1198

Powell, C., Beall, G., 2006. Physical properties of polymer/clay nanocomposites, Curr. Opin. Solid State Mater. Sci. 10, 73-80.

Ray, S., Okamoto, M., 2003. Polymer/layered silicate nanocomposites: a review from preparation to processing, Prog. Polym. Sci. 28, 1539-1641.

Rehab, A., Salahuddin, N., 2005. Nanocomposite materials based on polyurethane intercalated into montmorillonite clay, Mater. Sci. Eng. A 399, 368-376. 
Tahani, A., Karroua, M., Van Damme, H., Levitz, P., Bergaya, F., 1999. Adsorption of a cationic surfactant on Na-montmorillonite: Inspection of adsorption layer by X-Ray and fluorescence spectroscopies, J. Colloid Interface Sci. 216, 242-249.

Tiwari, R., Khilar, K., Natarajan, U., 2008. Synthesis and characterization of novel organomontmorillonites, Appl. Clay Sci. 38, 203-208.

Utracki, L., 2004. Clay-containing polymeric nanocomposites, volume 1, Rapra Technology Limited, Shawbury.

Vaccari, A., 1998. Preparation and catalytic properties of cationic and anionic clays, Catal. Today 41, 53-71.

Vaia, R., Teukolsky, R., Giannelis, E., 1994. Interlayer structure and molecular environment of alkylammonium layered silicates, Chem. Mater. 6, 1017-1022.

Wagener, R., Reisinger, T., 2003. A rheological method to compare the degree of exfoliation of Nanocomposites. Polymer 44, 7513-7518.

Wang, J., Calhoun, M., Severtson, S., 2008. Dynamic rheological study of paraffin wax and its organoclay nanocomposites. J. Appl. Polym. Sci., 108, 2564-2570.

Wang, S., Hu, Y., Zong, R., Tang, Y., 2004. Preparation and characterization of flame retardant ABS/montmorillonite nanocomposites, Appl. Clay Sci. 25, 49-55.

Xie, W., Gao, Z., Liu, K., Pan, W., Vaia, R., Hunter, D., Singh, A., 2001. Thermal characterization of organically modified montmorillonite, Termochim. Acta 367-368, 339-350. 
Yang, Y., Zhu, Z., Yin, J., Wang, X., Zong-Eng, Q., 1999. Preparation and properties of hybrids of organo-soluble polyimide and montmorillonite with various chemical surface modification methods, Polymer 40, 4407-4414.

Yui, T., Yoshida, H., Tachibana, H., Tryk, D., Inoue, H., 2002. Intercalation of polyfluorinated surfactants into clay minerals and the characterization of the hybrid compounds, Langmuir 18, 891-896.

Zhu, R., Zhu, L., Zhu, J., Xu, L., 2008. Structure of cetyltrimethylammonium intercalated hydrobiotite, Appl. Clay Sci. 42, 224-231. 
Table 1. Chemical composition (dry basis expressed as mass \%) of Boane bentonite samples before and after organic modification

\begin{tabular}{lcccccccccc}
\hline Sample & $\mathrm{SiO}_{2}$ & $\mathrm{Al}_{2} \mathrm{O}_{3}$ & $\mathrm{Fe}_{2} \mathrm{O}_{3}$ & $\mathbf{M g O}$ & $\mathrm{Na}_{2} \mathrm{O}$ & $\mathrm{CaO}$ & $\mathrm{K}_{2} \mathrm{O}$ & $\mathrm{P}_{2} \mathrm{O}_{5}$ & $\mathrm{TiO}_{2}$ & $\mathrm{MnO}$ \\
\hline Crude Boane & 80.16 & 13.16 & 2.70 & 2.66 & 0.85 & 0.14 & 0.08 & 0.00 & 0.22 & 0.03 \\
SC14 & 82.77 & 12.66 & 2.49 & 1.75 & 0.03 & 0.00 & 0.05 & 0.01 & 0.22 & 0.01 \\
SC14+DC16 & 82.58 & 12.66 & 2.51 & 1.94 & 0.05 & 0.00 & 0.05 & 0.02 & 0.21 & 0.00 \\
\hline
\end{tabular}

Table 2. Estimating organic content from TG results.

\begin{tabular}{lccccc}
\hline Sample & \multicolumn{2}{c}{ Mass loss, mass \% } & \multicolumn{3}{c}{ Organic content, mass \% } \\
& $\mathbf{1 5 0}^{\mathbf{}} \mathbf{C}$ & $\mathbf{1 0 0 0}^{\mathbf{C}} \mathbf{C}$ & Theory $^{\mathbf{1}}$ & Experimental & \% CEC \\
\hline Crude & 83.4 & 79.3 & - & - & - \\
Slurry & 19.2 & 18.0 & - & - & - \\
SC14 & 97.1 & 71.9 & 19.0 & 20.9 & 110 \\
SC14+DC16 & 97.9 & 60.5 & 28.1 & 34.0 & 121 \\
DC16 & 98.1 & 55.8 & 35.3 & 39.3 & 111 \\
\hline
\end{tabular}

${ }^{1}$ Based on the assumption of CEC level exchange 
Table 3. Position of the FTIR $v_{\text {as }}\left(\mathrm{CH}_{2}\right)$ band and the basal spacings

\begin{tabular}{ccc}
\hline Sample & FTIR vas $\left(\mathbf{C H}_{2}\right)$ & XRD $\mathbf{d}$-spacing \\
\hline Crude & $\mathbf{c m}^{-\mathbf{1}}$ & $\mathbf{n m}$ \\
SC14 & - & 1.50 \\
SC14+DC16 & 2923 & 2.00 \\
DC16 & 2924 & 3.68 \\
\hline
\end{tabular}

Table 4. Wear and friction coefficient results from the High-Frequency Reciprocating Rig apparatus

\begin{tabular}{lccccc|}
\hline Sample & White oil & SC14 & DC16 & SC14+DC16 & $\begin{array}{c}\text { SC14+DC16 } \\
\text { (Koppies) }\end{array}$ \\
\hline Mean wear scar, $\mu \mathrm{m}$ & 165 & 480 & 555 & $565 \& 585$ & 595 \\
Friction coefficient (mean) & 0.07 & 0.13 & 0.12 & $0.13 \& 0.14$ & 0.13 \\
\hline
\end{tabular}




\section{List of Figures}

Fig. 1. SEM/EDS image of organo-bentonite SC14+DC16 embedded in epoxy resin.

Fig. 2. FESEM micrographs of crude bentonite [A] and organo-bentonite SC14 [B].

Fig. 3. TG curves of untreated and organically treated bentonites

Fig. 4. DTA curves of crude and organically treated bentonites

Fig. 5. XRD diffractograms of untreated and organically treated bentonites

Fig. 6. FT-IR spectra of raw and organo-modified bentonites

Fig. 7. Effect of the shear rate on the viscosities of white oil and white oil dispersions containing 10 mass $\%$ organo-bentonite (inorganic basis). $\diamond$ : white oil; $\Delta$ : SC14; $\square$ : SC14+DC16; o: DC16. 


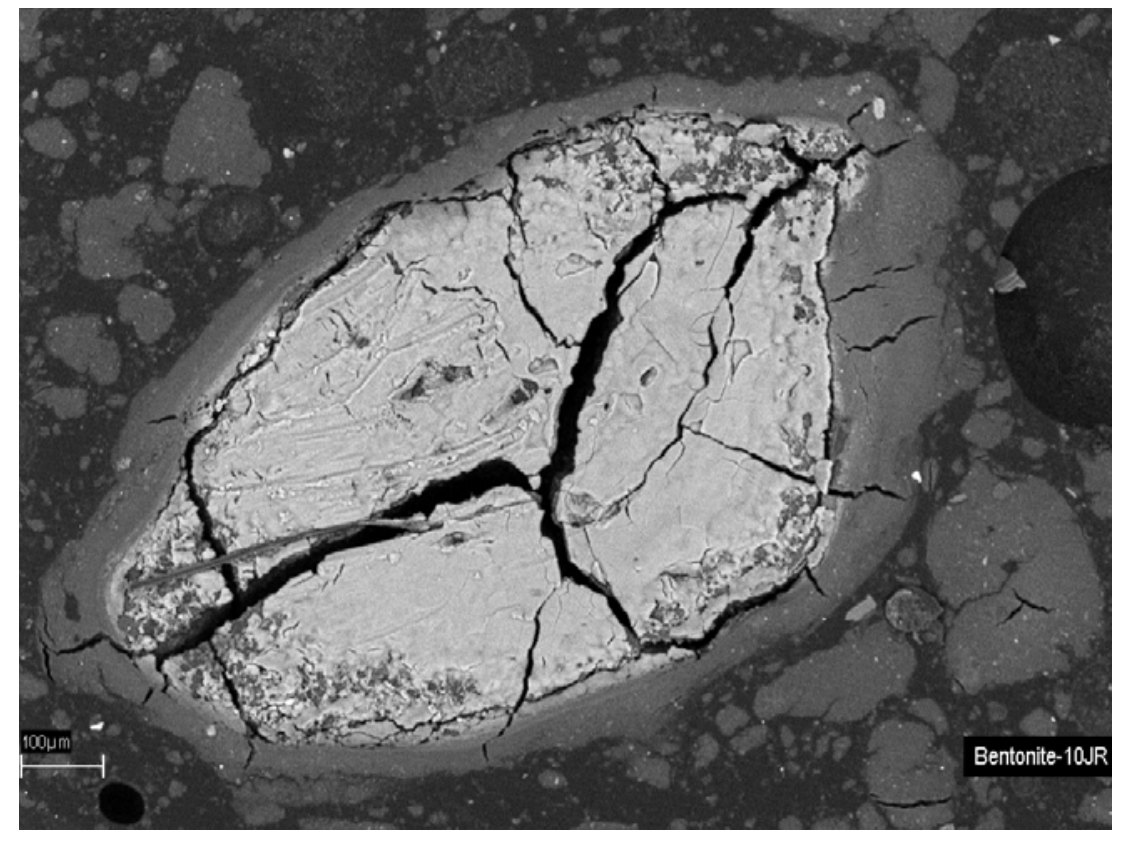

Fig. 1. SEM/EDS image of organo-bentonite SC14+DC16 embedded in epoxy resin. 


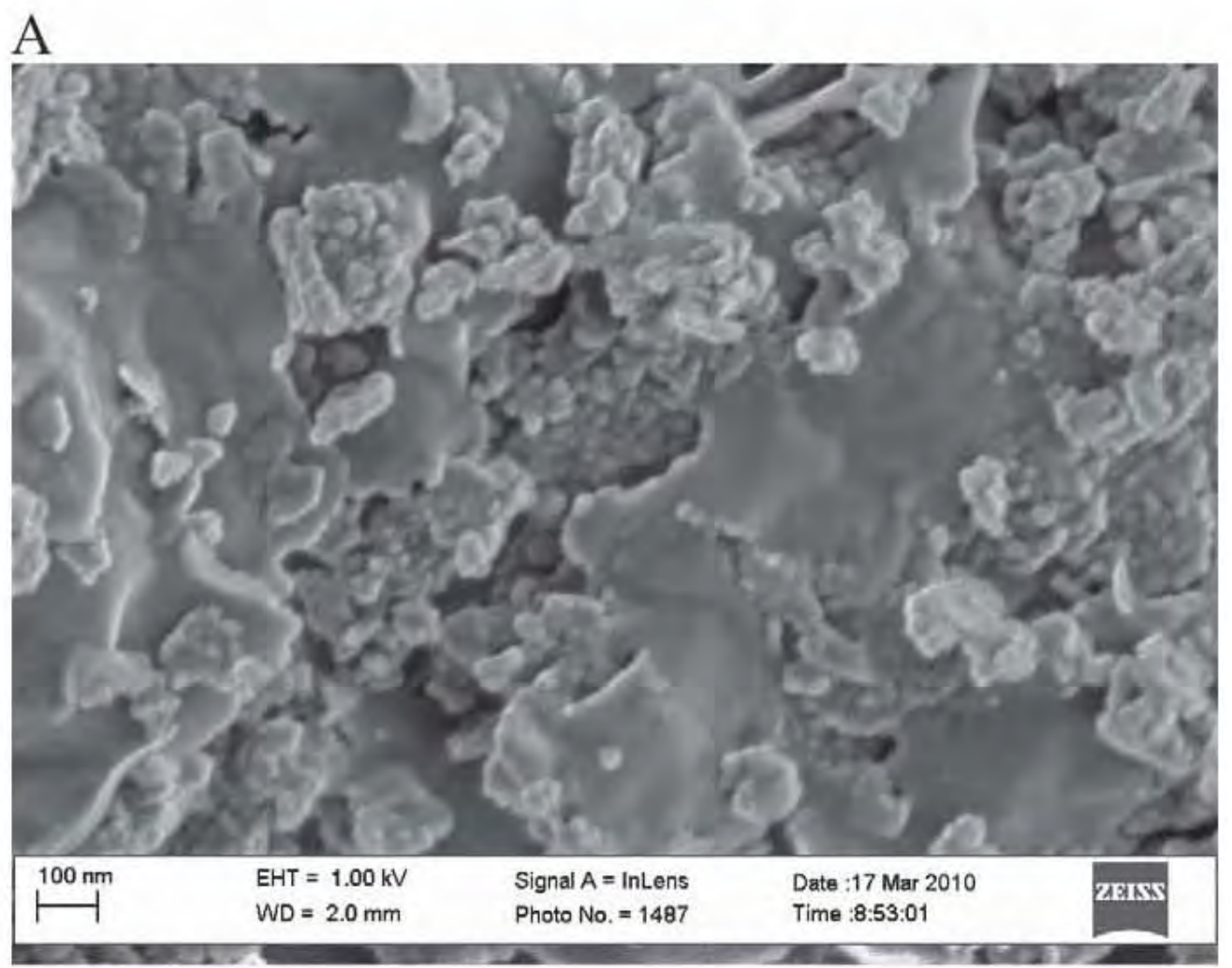

B

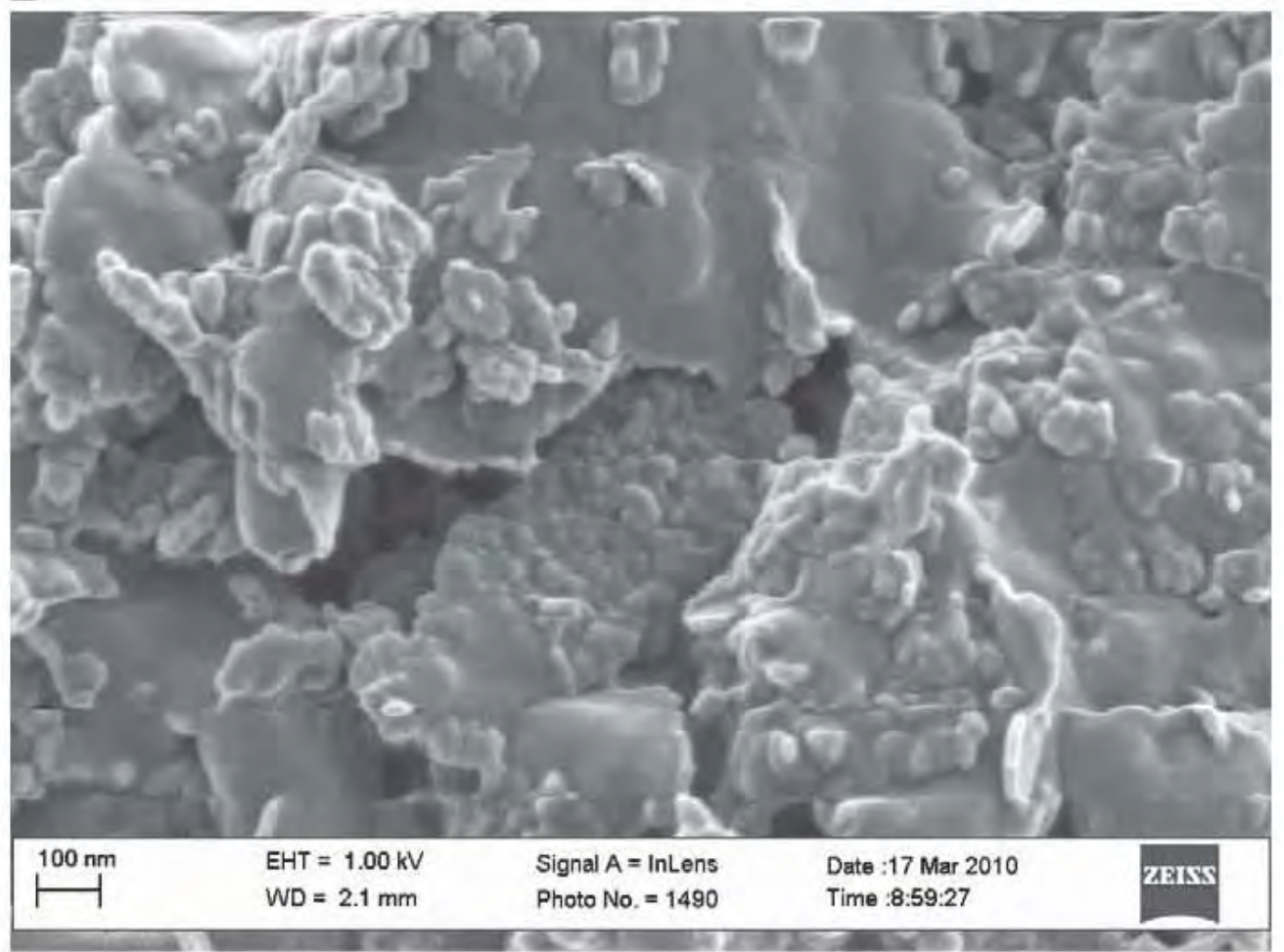

Fig. 2. FESEM micrographs of crude bentonite [A] and organo-bentonite SC14 [B]. 


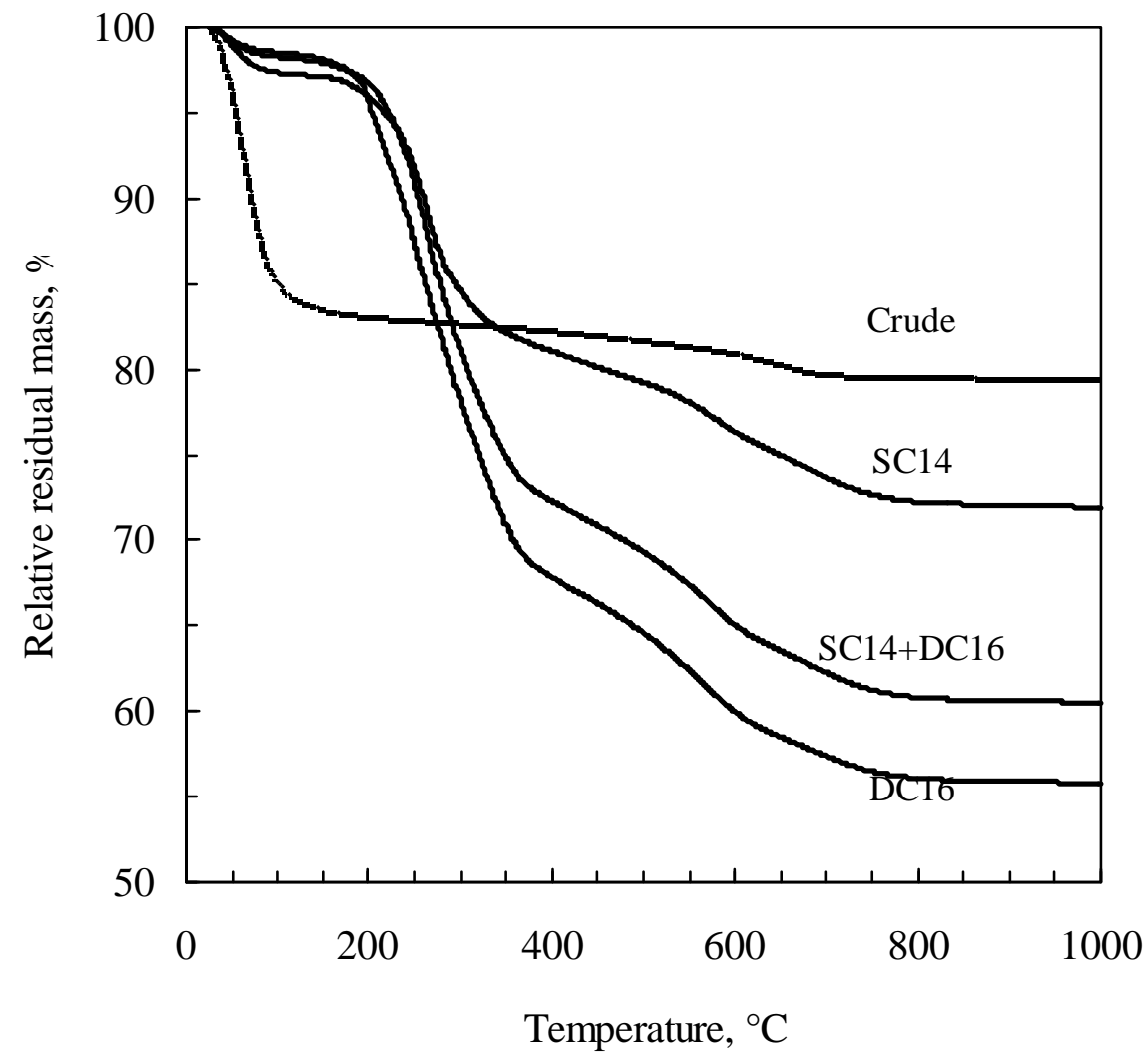

Fig. 3. TG curves of untreated and organically treated bentonites 


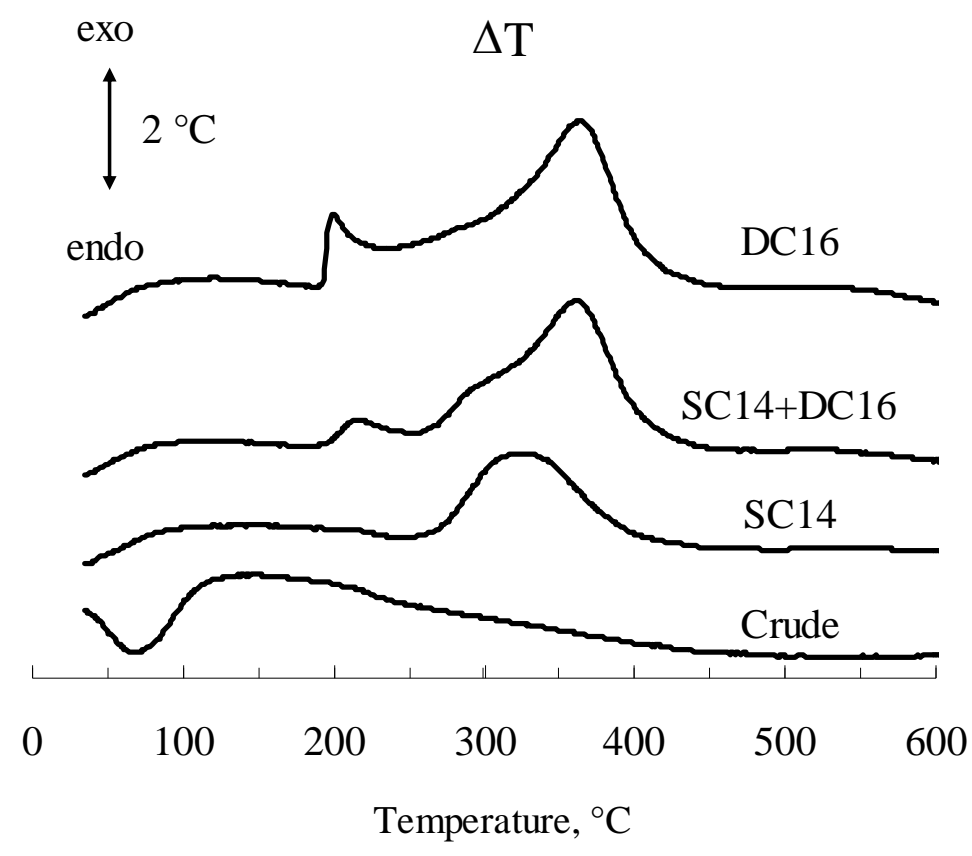

Fig. 4. DTA curves of crude and organically treated bentonites 


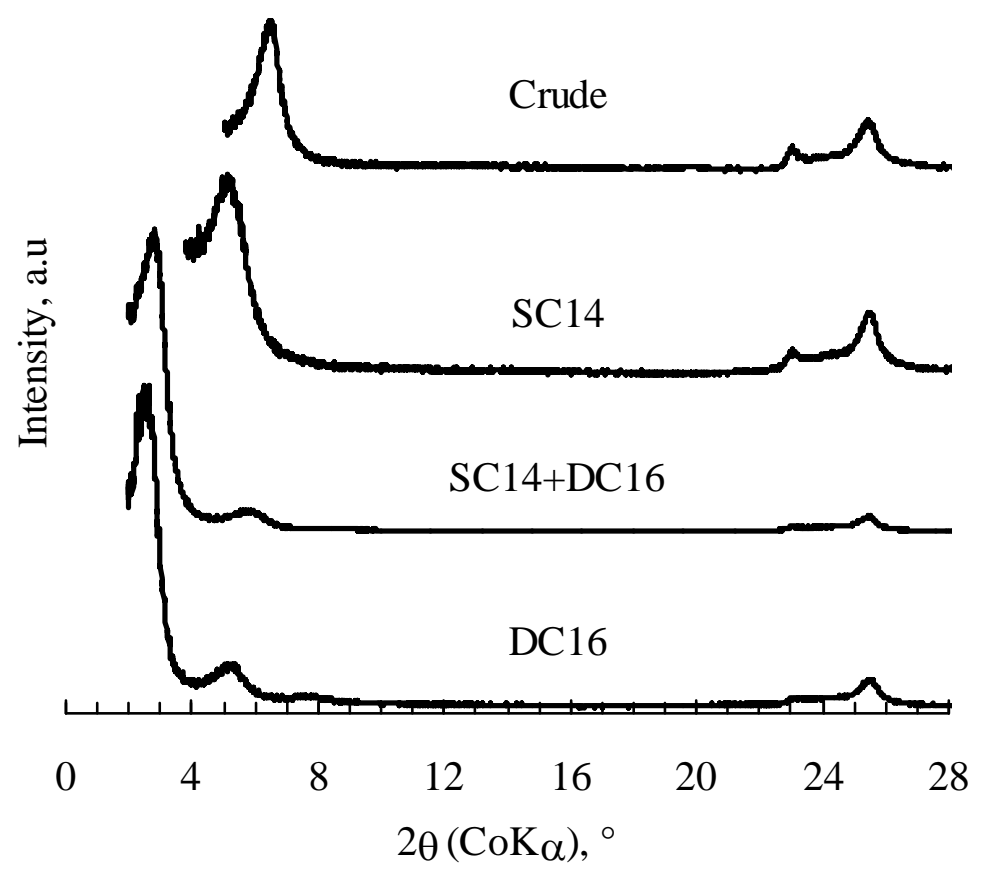

Fig. 5. XRD diffractograms of untreated and organically treated bentonites 


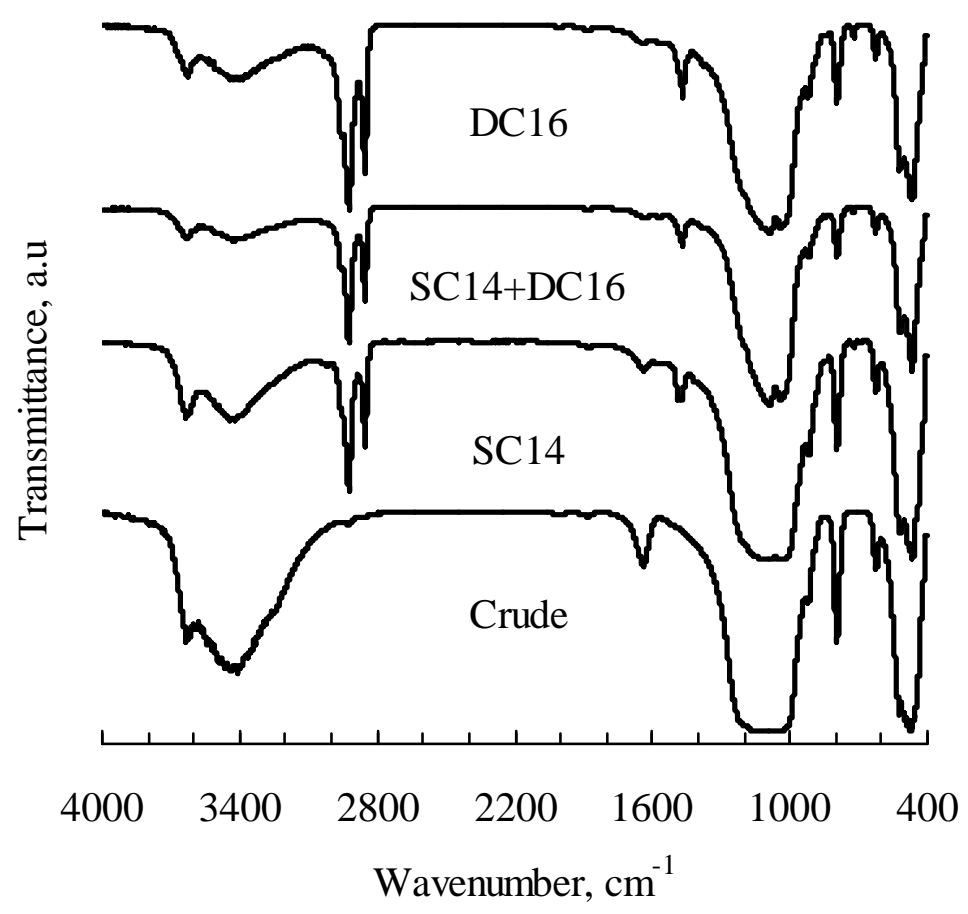

Fig. 6. FT-IR spectra of raw and organo-modified bentonites 


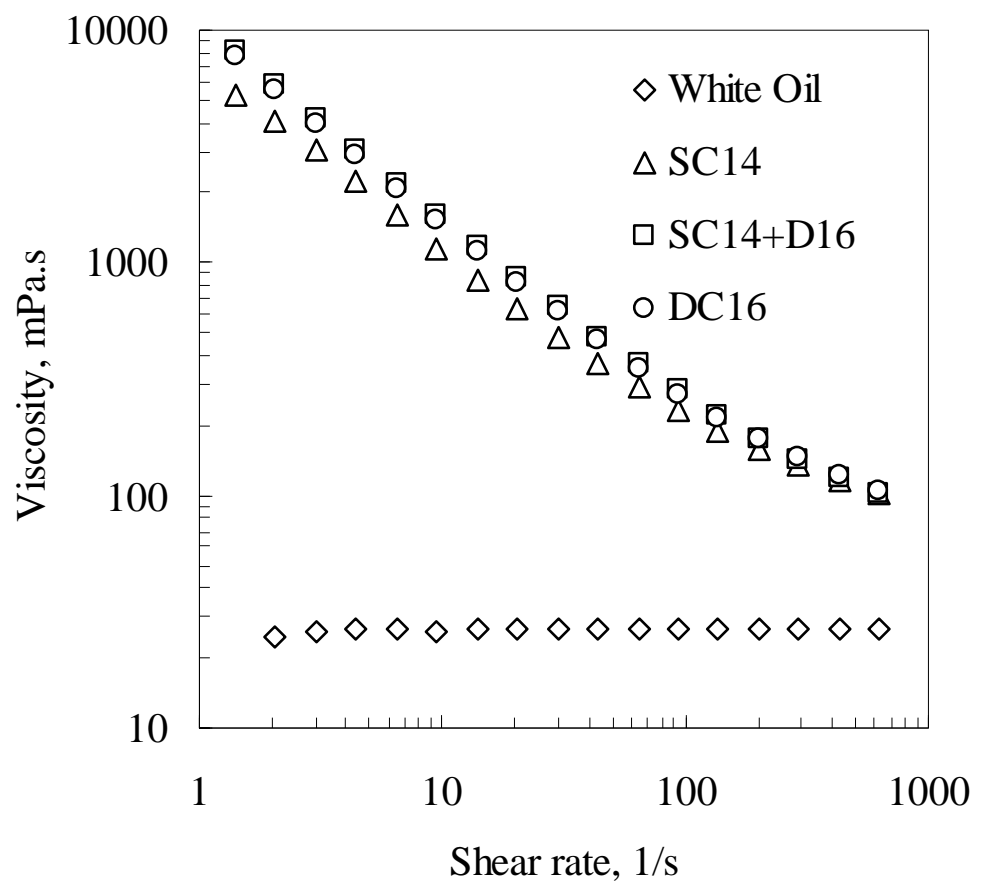

Fig. 7. Effect of the shear rate on the viscosities of white oil and white oil dispersions containing 10 mass $\%$ organo-bentonite (inorganic basis). $\diamond$ : white oil; $\Delta$ : SC14; $\square$ : SC14+DC16; o: DC16. 